\title{
Gelatin microparticles aggregates as three-dimensional scaffolding system in cartilage engineering
}

\author{
D. M. García Cruz $\cdot$ V. Sardinha · J. L. Escobar Ivirico • \\ J. F. Mano · J. L. Gómez Ribelles
}

Received: 2 July 2012/ Accepted: 8 November 2012/Published online: 18 November 2012

(C) Springer Science+Business Media New York 2012

\begin{abstract}
A three-dimensional (3D) scaffolding system for chondrocytes culture has been produced by agglomeration of cells and gelatin microparticles with a mild centrifuging process. The diameter of the microparticles, around $10 \mu$, was selected to be in the order of magnitude of the chondrocytes. No gel was used to stabilize the construct that maintained consistency just because of cell and extracellular matrix (ECM) adhesion to the substrate. In one series of samples the microparticles were charged with transforming growth factor, TGF- $\beta 1$. The kinetics of growth factor delivery was assessed. The initial delivery was approximately $48 \%$ of the total amount delivered up to day 14. Chondrocytes that had been previously expanded in monolayer culture, and thus dedifferentiated, adopted in this $3 \mathrm{D}$ environment a round morphology, both with presence or absence of growth factor delivery, with
\end{abstract}

D. M. García Cruz · V. Sardinha · J. L. Escobar Ivirico ( $\square)$.

J. L. Gómez Ribelles

Center for Biomaterials and Tissue Engineering, Universitat

Politècnica de València, Camino de Vera s/n, 46022 Valencia, Spain

e-mail: joresciv@ter.upv.es

V. Sardinha $\cdot$ J. F. Mano

3B's Research Group - Biomaterials, Biodegradables and

Biomimetics, University of Minho, Headquarters of the

European Institute of Excellence on Tissue Engineering and

Regenerative Medicine, AvePark, S. Claúdio do Barco,

Taipas 4806-909, Guimarães, Portugal

V. Sardinha $\cdot$ J. F. Mano

ICVS/3B's - PT Government Associate Laboratory, Braga/

Guimarães, Portugal

J. L. Gómez Ribelles

Networking Research Center on Bioengineering, Biomaterials and Nanomedicine (CIBER-BBN), Valencia, Spain production of ECM that intermingles with gelatin particles. The pellet was stable from the first day of culture. Cell viability was assessed by MTS assay, showing higher absorption values in the cell/unloaded gelatin microparticle pellets than in cell pellets up to day 7. Nevertheless the absorption drops in the following culture times. On the contrary the cell viability of cell/TGF- $\beta 1$ loaded gelatin microparticle pellets was constant during the 21 days of culture. The formation of actin stress fibres in the cytoskeleton and type I collagen expression was significantly reduced in both cell/gelatin microparticle pellets (with and without TGF- $\beta 1$ ) with respect to cell pellet controls. Total type II collagen and sulphated glycosaminoglycans quantification show an enhancement of the production of ECM when TGF- $\beta 1$ is delivered, as expected because this growth factor stimulate the chondrocyte proliferation and improve the functionality of the tissue.

\section{Introduction}

A three-dimensional (3D) arrangement of cells in an adequate substrate seems to be a requirement for the development and maintenance of the phenotype of hyaline cartilage chondrocytes [1]. Mature chondrocytes can be isolated from the cartilage tissue obtained in a biopsy of articular cartilage. When they are plated in monolayer culture on plastic substrates, they can grow to the cell numbers required for tissue regeneration but they loose their native phenotype showing clear changes in cell morphology and expression of type I collagen which is nearly absent in articular cartilage tissue [2]. The re-differentiation of these cells in 3D cultures is thus a subject of great interest for cartilage engineering. 
A huge number of different 3D scaffolding systems have been proposed for cartilage engineering including sponges, fibber meshes, gels and microparticles [3] with varying materials of synthetic or natural origin and a variety of pore architecture. Among them, 3D arrangements obtained by fusion of microparticles as scaffolding blocks have been investigated [3]. The drawback of these systems is that global porosity is not high enough for cell survival, and the strong linkage between particles allows few spaces for production and accumulation of ECM components. On the other hand, the interest in developing injectable scaffolding systems yielded to explore a number of combinations of synthetic microparticles and "in situ" crosslinkable hydrogels [4-8].

Gelatin microparticles (Gel-MCP) have been explored extensively as drug or growth factor delivery systems. In particular they have been used for delivery of TGF- $\beta 1$ or TGF- $\beta 3$ growth factors in "in vitro" chondrogenic differentiation of bone marrow mesenchymal stem cells. TGF- $\beta 3$ loaded Gel-MCP were mixed with cells to form a pellet by centrifugation, labelling as a modified pellet by Fan et al. [9]. A small amount of microparticles was used in that work in order to preserve the large cell to cell contact that favours chondrogenic differentiation of mesenchymal stem cells. Han et al. [10] used a mixture of gelatin and chitosan microparticles with the same purpose, while Park et al. [11] used a hydrogel to entrap both cells and Gel-MCP. A similar construct was proposed by $\mathrm{Hu}$ et al. [12] who introduced microparticles and chondrocytes in a chitosan hydrogel. Gelatin has been also used to functionalize microparticles made of synthetic biodegradable polyester such as polylactide/polyglycolide copolymers, PLGA, [13].

On the other hand Gel-MCP have been used as microcarriers for chondrocyte expansion in stirring bioreactors. It was shown that human nasal chondrocytes expanded on the surface and in the micropores of the microcarriers maintain the ability to differentiate to hyaline cartilage chondrocytes in pellet culture [14]. These microcarriers have been used in spinner flasks as well [15, 16]. Gelatin has been also used to fabricate macroporous scaffolds for cartilage engineering profiting the capacity of delivering growth factors from the scaffold itself [17]. Although the mentioned works show that gelatin is a suitable substrate for chondrocyte $3 \mathrm{D}$ culture none has proved to be the ultimate solution. Results obtained from tissue engineering procedures suggest that the proposed gelatin scaffolding systems may lead to a mixture of hyaline and fibrocartilage tissue.

In our work we explore the use of Gel-MCP not only as a growth factor delivery vehicle but also as a 3D scaffolding system. Dedifferentiated chondrocytes are mixed with microparticles at a Gel-MCP/cells ratio high enough to form a construct in which cells are surrounded by the gelatin as the scaffolding material. In addition in one of the experimental series Gel-MCP will be loaded with TGF- $\beta 1$ which is delivered continuously during "in vitro" culture. It will be shown that Gel-MCP can agglomerate in situ by the action of the cells; the particles thus provide a 3D structural support for cells which is able to release relevant bioactive molecules at the same time.

\section{Materials and methods}

\subsection{Preparation of unloaded and TGF- $\beta 1$ loaded Gel- MCP}

Crosslinked Gel-MCP encapsulating TGF- $\beta 1$ growth factor were prepared by emulsification solvent extraction and crosslinking method [18]. Briefly, $1 \mathrm{~g}$ of gelatin (Scharlab, Spain) was dissolved in $10 \mathrm{~mL}$ of Dulbecco's phosphate buffer solution (DPBS) at $50{ }^{\circ} \mathrm{C}$ to a final concentration of 10 wt. \%. Subsequently, gelatin solution was cooled to $37{ }^{\circ} \mathrm{C}$, mixed with the TGF- $\beta 1$ (Sigma, Spain) solution $(20 \mu \mathrm{g} / \mathrm{mL})$ and homogenized. Then, the mixture was added into olive oil at a feeding rate of $1 \mathrm{~mL} / \mathrm{min}$ under stirring at $1,500 \mathrm{rpm}$ for $30 \mathrm{~min}$. After this time, $10 \mathrm{~mL}$ of $20 \mathrm{mM}$ genipin (Wako Chemical, Japan) solution was added into the biphasic system at a rate of $1 \mathrm{~mL} / \mathrm{min}$ and maintained under stirring for $17 \mathrm{~h}$. Finally, loaded crosslinked Gel-MCP were collected by centrifugation, washed several times in acetone to remove residual olive oil and lyophilised. The loaded microparticles were stored at $-20{ }^{\circ} \mathrm{C}$ until further use. Unloaded crosslinked Gel-MCP were also prepared and used as reference. The percentage yield of the lyophilized TGF- $\beta 1$ loaded Gel-MCP crosslinked with genipin was calculated dividing the weight of microparticles obtained by initial weight of gelatin used. The unloaded Gel-MCP were obtained using the same procedure, without add TGF- $\beta 1$ in the gelatin solution.

\subsection{Determination of crosslinking degree}

Ninhydrin (NHN) assay [19] was used to determine the percentage of free amino groups remaining in the Gel-MCP after crosslinking reaction. Ninhydrin (Sigma-Aldrich, Spain) solution was freshly prepared on the day of the assay dissolving $0.8 \mathrm{~g}$ of NHN and $0.12 \mathrm{~g}$ of hydrindantin (Sigma-Aldrich, Spain) in $40 \mathrm{~mL}$ of ethylene glycol (Sigma-Aldrich, Spain). This solution was then mixed with lithium acetate buffer (4 M, pH 5.2) to prepare the working reagent. For the assay, $0.5 \mathrm{mg}$ of lyophilized Gel-MCP (uncrosslinked and crosslinked with genipin) was mixed with $0.5 \mathrm{~mL}$ of working reagent in eppendorf tubes. Then, the tubes were immediately capped, shaken and heated to 
$100{ }^{\circ} \mathrm{C}$ in a water bath for $30 \mathrm{~min}$ to allow the reaction to proceed. The solution was then cooled down to room temperature, diluted with $5 \mathrm{~mL}$ of $50 \%$ isopropanol and vortexed for $15 \mathrm{~s}$ in order to oxidise the excess of hydrindantin. The absorbance of each solution was measured at $570 \mathrm{~nm}$ using a UV spectrophotometer (Cecil CE9200, UK). The amount of free amino groups in the assayed microparticles was determined by a calibration curve of glycine $(n=5)$. The crosslinking degree was calculated according to the Eq. 1.

$$
\begin{aligned}
& \text { Crosslinking degree }= \\
& \frac{\left[(\text { NHN reactive amine })_{\text {fresh }}-(\text { NHN reactive amine })_{\text {fixed }}\right]}{(\text { NHN reactive a Mine })_{\text {fresh }}} * 100
\end{aligned}
$$

where, "fresh" is the mole fraction of free $\mathrm{NH}_{2}$ groups in the uncrosslinked and unloaded Gel-MCP and "fixed" is the mole fraction of free $\mathrm{NH}_{2}$ groups remaining in the unloaded Gel-MCP after the crosslinked reaction with genipin.

\subsection{Determination of encapsulation efficiency (EE)}

The EE of the TGF- $\beta 1$ loaded Gel-MCP crosslinked with genipin was expressed as the percentage of the TGF- $\beta 1$ amount in the microparticles with respect to the initial TGF- $\beta 1$ used. For that, $2 \mathrm{mg}$ of TGF- $\beta 1$ loaded Gel-MCP were swelled in DPBS and centrifuged at 15,000 rpm for $30 \mathrm{~min}$, three times, in order to extract the TGF- $\beta 1$ from the Gel-MCP $(n=5)$. The supernatant was extracted and the TGF- $\beta 1$ was quantified by an enzyme-linked immunosorbent assay kit (R\&D systems, USA).

\subsection{Morphological analysis}

The morphology of unloaded gelatin and TGF- $\beta 1$ loaded Gel-MCP were examined by scanning electron microscopy (SEM) (Jeol JSM-5410, Japan). All samples were coated with a conductive layer of sputtered gold. The micrographs were taken at an accelerating voltage of $15 \mathrm{kV}$ in order to ensure a suitable image resolution. Microparticle size distribution was determined analysing 10 different images, with around 30 microparticles in each one.

\subsection{Water uptake studies}

The water uptake of Gel-MCP was determined by immersion in distilled water at $37{ }^{\circ} \mathrm{C}$ for $24 \mathrm{~h}$. The weight of the dry and swollen microparticles was measured and the water uptake (W) was calculated using the following equation:
$W=$

$\frac{(\text { weight of swollen Gel }-M C P-\text { weight of dry Gel }-M C P)}{\text { weight of dry Gel }- \text { MCP }} * 100$

Uncrosslinked microparticles were also prepared and were used as control in the water uptake studies $(n=5)$. The appearance of swollen crosslinked and uncrosslinked Gel-MCP were observed using an optical microscope (ECLIPSE-E6000, Nikon, Japan).

\subsection{In vitro release studies}

The in vitro release profile of TGF- $\beta 1$ from Gel-MCP was examined over 14 days. The microparticles were dispersed into $1 \mathrm{~mL}$ of DPBS (pH 7.4). These dispersions were immediately placed in an orbital shaker water bath at $37{ }^{\circ} \mathrm{C}$ and were shaken at a frequency of $60 \mathrm{rpm}$. After 0.04, 0.08, $0.125,0.16,0.20,1,2,4,7,10$ and 14 days, the microparticle suspension was centrifuged at 5,000 rpm to collect the supernatant for analysis $(n=8)$. The amount of TGF$\beta 1$ in the supernatant was measured using an enzymelinked immunosorbent assay kit (eBioscience, USA).

\subsection{Cell isolating and seeding}

Chondrocytes were isolated from human articular cartilage from the knee of a patient undergoing total knee arthroplasty by standard protocols [20] and in accordance with the Declaration of Helsinki of 1975 as revised in 1983, and approved by our local Ethical Committee. All subjects provided a written informed consent before their inclusion in the study. The chondrocytes used in this study were supplied by IMIM (Institut Municipal d'Investigació Mèdica, Barcelona, Spain). Cells were plated in culture flasks (Nunc, Spain) at high density in Dulbecco's Modified Eagle's Medium (DMEM) supplemented with $10 \%$ fetal bovine serum (FBS) (both reagents supplied by Gibco, UK) and $50 \mu \mathrm{g} / \mathrm{mL}$ ascorbic acid (Sigma-Aldrich, Spain) at $37{ }^{\circ} \mathrm{C}$ in a $5 \% \mathrm{CO}_{2}$ humidified atmosphere. The culture medium was renewed every 2-3 days. After 5 days, adherent cells were enzymatically harvested with tripsin/ EDTA (Gibco, UK) and seeded on the Gel-MCP, as control sample, and TGF- $\beta 1$ loaded Gel-MCP at cell density of $2.5 \times 10^{5}$ cells/tubes. To obtain the cell/Gel-MCP pellets (with and without TGF- $\beta 1$ ), tubes that contained $20 \mathrm{mg}$ of loaded or unloaded Gel-MCP and chondrocytes were centrifuged at $300 \mathrm{~g} \times 5 \mathrm{~min}$. Cell pellets without any sample were used as reference. The experiments were conducted for 3 weeks in chondrogenic media consisting of Dulbecco's high glucose modified Eagle medium with $1 \%$ ITS + Premix (DB Biosciences, USA), $100 \mu \mathrm{g} / \mathrm{mL}$ sodium pyruvate (Gibco, UK), $1 \%$ L-glutamine (Gibco, UK), 
$100 \mu \mathrm{g} / \mathrm{mL}$ penicillin-streptomycin (Lonza, USA) supplemented with $50 \mu \mathrm{g} / \mathrm{mL}$ ascorbic acid (Sigma-Aldrich, Spain). The culture medium was replaced three times/week.

\subsection{Cell adhesion and morphology}

Chondrocyte adhesion and morphology were investigated by scanning electron microscopy, SEM (Jeol JSM-5410, Japan). For this purpose, cell/gel-MCP pellets (with and without TGF- $\beta 1$ ) and cell pellets samples were removed from the culture medium at different times $(1,7,14$ and 21 days), washed in PBS, fixed in $2.5 \%$ glutaraldehyde, rinsed twice with PBS and dehydrated in series of ethanol solution $(30,50,70,90 \%$ and absolute) for $15 \mathrm{~min}$ with final dehydration in absolute ethanol for $30 \mathrm{~min}$. Finally, samples were dried at room temperature and sputter coated with gold using a Fisons Instrument Coater (Polaron SC 502, UK) before observation with SEM.

\subsection{Cell viability}

The viability of chondrocytes cultured for $1,7,14$ and 21 days was determined using the MTS (3-(4,5-dimethylthiazol-2-yl)-5-(3-carboxymethoxyphenyl)-2-(4-sulfophenyl)$2 \mathrm{H}$-tetrazolium) assay following manufacturer instructions (Promega, Spain). The samples were washed twice with PBS and incubated with fresh culture medium without phenol red and FBS containing MTS reagent (ratio 5:1) at $37^{\circ} \mathrm{C}$ for $3 \mathrm{~h}$ in darkness. After the incubation period, the absorbance was quantified by spectrophotometry at $490 \mathrm{~nm}$ with a microplate reader (VICTOR3TM, PerkinElmer, USA). The background absorbance values obtained from unseeded gelatin microparticle pellets (with and without TGF- $\beta 1$ ) were subtracted to the seeded pellets absorbance values to get the final viable cell number.

\subsection{Immunofluorescence}

Aggrecans and type I collagen were detected by immunofluorescence assays. Cell/Gel-MCP pellets (with and without TGF- $\beta 1$ ) with $8 \mathrm{~mm}$ of diameter were fixed with formalin and sectioned in slices $(\sim 2 \mathrm{~mm})$, washed with PBS twice and permeabilized with $0.1 \%$ v/v Triton X-100 in PBS. The cell pellet of $1 \mathrm{~mm}$ of diameter was fixed and washed as previously described. Afterwards, all samples were incubated with $10 \%$ fetal bovine serum in PBS blocking solution for $2 \mathrm{~h}$ at room temperature. Then, samples were incubated in the dilute primary antibody solution (Mouse anti-Human Aggrecan and Rabbit antihuman Type I Collagen, 1:50, from Invitrogen, Spain and Chemicon, Spain respectively) overnight at $4{ }^{\circ} \mathrm{C}$, samples were washed again three times in PBS and then incubated with the respective secondary fluorochrome-conjugated antibody (1:200) for $2 \mathrm{~h}$ at room temperature in dark. Also, the formation of actin cytoskeleton was visualized using bodipy FL phallacidin (Invitrogen, Spain). Finally, samples were washed three times with PBS and mounted with a drop of mounting medium containing DAPI for nuclei staining (blue).

\subsection{Biochemical analysis}

The type II collagen secreted by the chondrocytes were determined quantitatively using type II Collagen Elisa kit (MD Bioproducts, USA) after pepsin digestion of cell/GelMCP pellets (with and without TGF- $\beta 1$ ) and cell pellets ( $n=5)$ according to the supplier's instructions. Using $500 \mu \mathrm{L}$ of digested solution, GAG content was quantified following instructions provided by the manufacturer in the Blyscan_Sulfated Glycosaminoglycan Assay kit (Biocolor Ltd., UK). Bound dye values were quantified at $656 \mathrm{~nm}$ using a microplate reader (VICTOR3TM, PerkinElmer, USA).

\subsection{Statistical analysis}

Data were expressed as mean values \pm standard deviation (SD) of the mean (mean $\pm \mathrm{SD}$ ) and analysed by one-way analysis of variance (ANOVA) and Student's $t$ test. The level of statistical significance was set as $P<0.05$.

\section{Results}

\subsection{Characterization of Gel-MCP}

The yield of Gel-MCP (with and without TGF- $\beta 1$ ) was around $90 \%$. The crosslinking degree through NHN assay was determined in order to know the quantities of free $\mathrm{NH}_{2}$ remaining in the Gel-MCP after the reaction with genipin. The obtained results indicate that a $74 \%$ free $\mathrm{NH}_{2}$ remain in the gelatin chemical structure.

The EE has a strong dependency with the preparation technique used to synthesize the microparticles. The TGF$\beta 1$ loaded Gel-MCP crosslinked with genipin retain more than $85 \pm 1 \%$ of the TGF- $\beta 1$ initially used.

The morphology of Gel-MCP (with and without TGFß1) was examined by SEM (Fig. 1). The microparticles showed a spherical morphology of all samples with slightly roughness surface without wrinkles or cracks. On the other hand, the microparticles have a homogeneous size, ranging from 6 to $24 \mu \mathrm{m}$ in diameter with a mean diameter size in both cases (microparticles with and without TGF- $\beta 1$ ) of $12 \mu \mathrm{m}$ (see Fig. 1). 
Fig. 1 Morphology and particle size distribution of crosslinked Gel-MCP without $(\mathbf{a}, \mathbf{c})$ and with (b, d) TGF- $\beta 1$
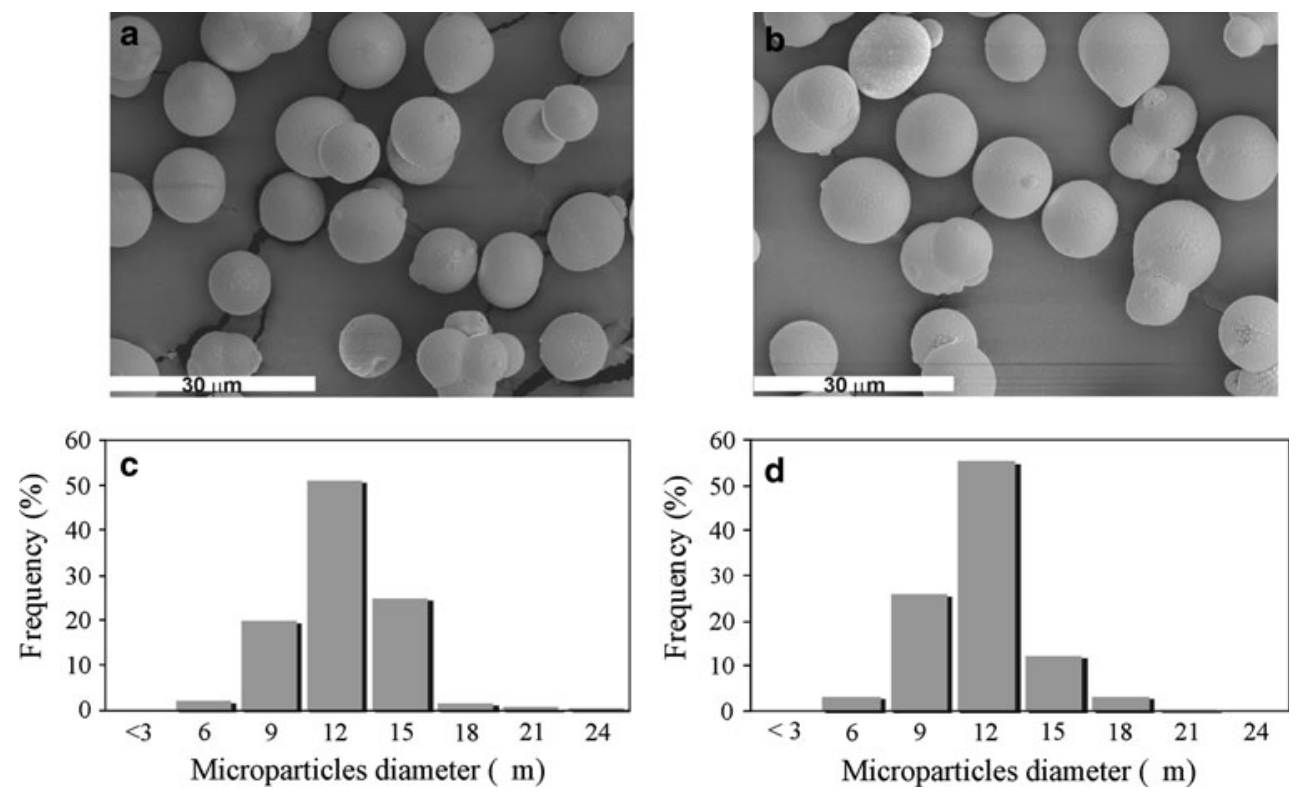

Dry state
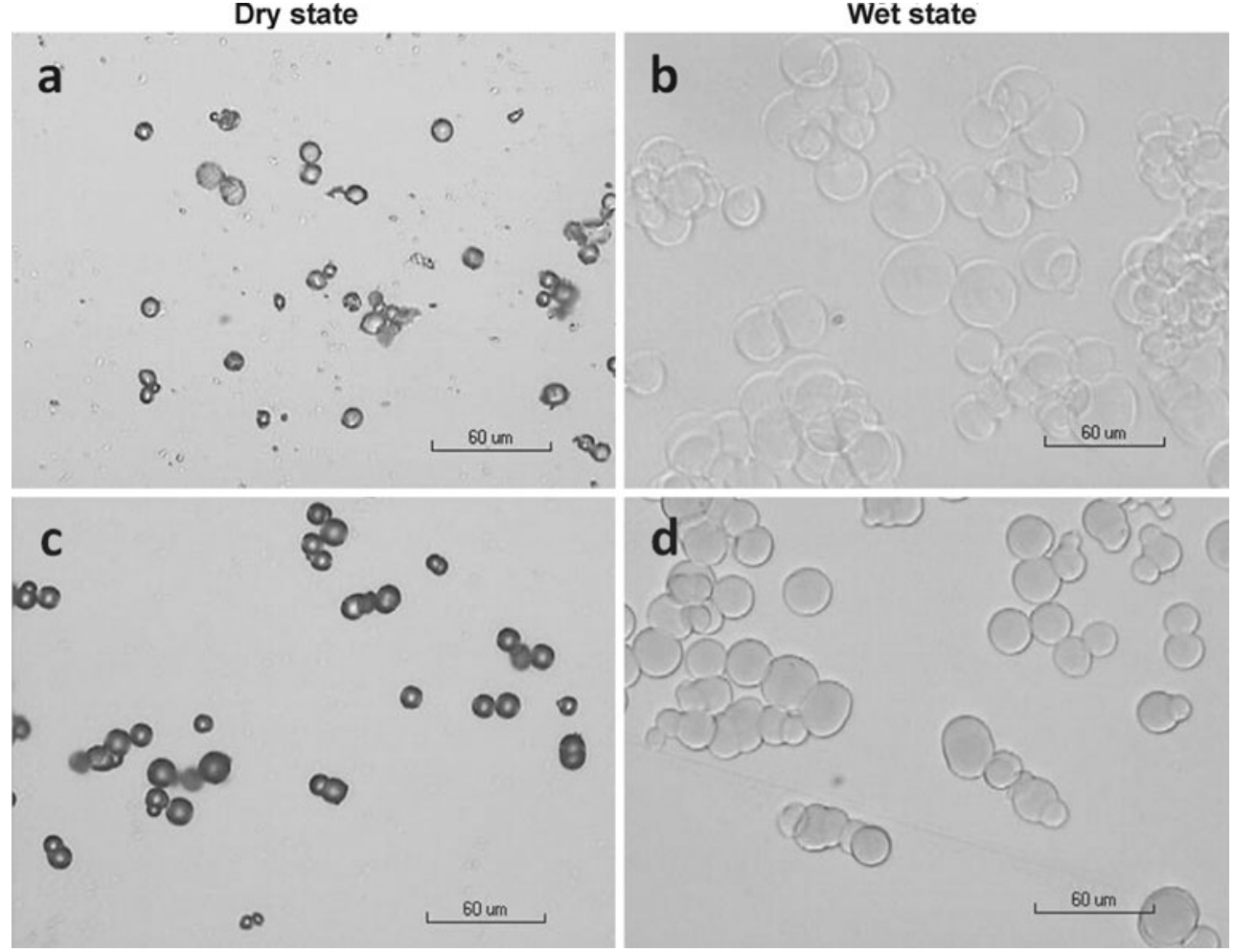

Fig. 2 Gross appearance of non-crosslinked $(\mathbf{a}, \mathbf{b})$ and crosslinked (c, d) Gel-MCP in dry and wet state

\subsection{Water uptake experiments}

Figure 2 shows the morphological appearance of the noncrosslinked (a,b) and crosslinked (c,d) Gel-MCP in dry and wet state. After $24 \mathrm{~h}$, the water uptake of the crosslinked Gel$\operatorname{MCP}(111.5 \pm 12.5 \%)$ significantly decrease compared with the values of the non-crosslinked ones $(325.3 \pm 11.1 \%)$.

\subsection{In vitro TGF- $\beta 1$ release study}

After the encapsulation process, $38.4 \mathrm{ng}$ of TGF- $\beta 1$ were loaded in $20 \mathrm{mg}$ of Gel-MCP. The accumulative release profile of TGF- $\beta 1$ from the Gel-MCP crosslinked with genipin over 14 days at $37{ }^{\circ} \mathrm{C}$ in D-PBS was represented in Fig. 3. TGF- $\beta 1$ release pattern showed a burst until 24 h, 


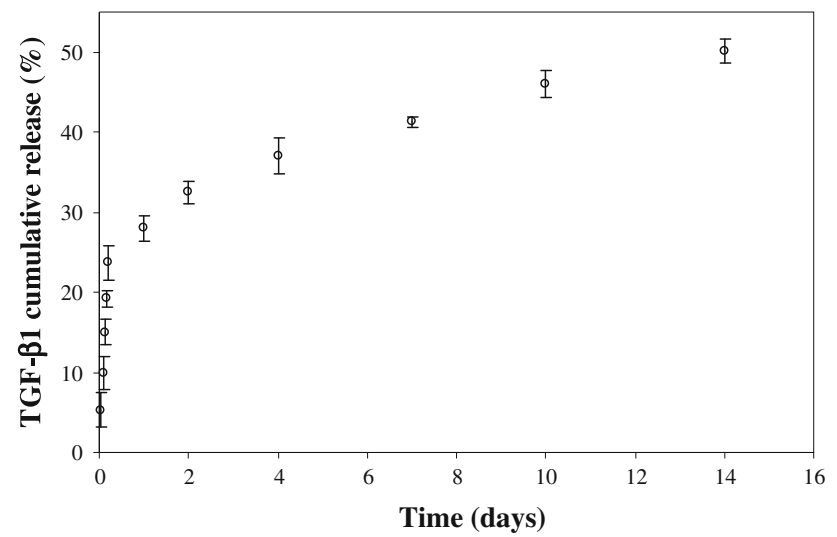

Fig. 3 Cumulative TGF- $\beta 1$ release profile from Gel-MCP. Error bars represent means \pm SD with $n=8$

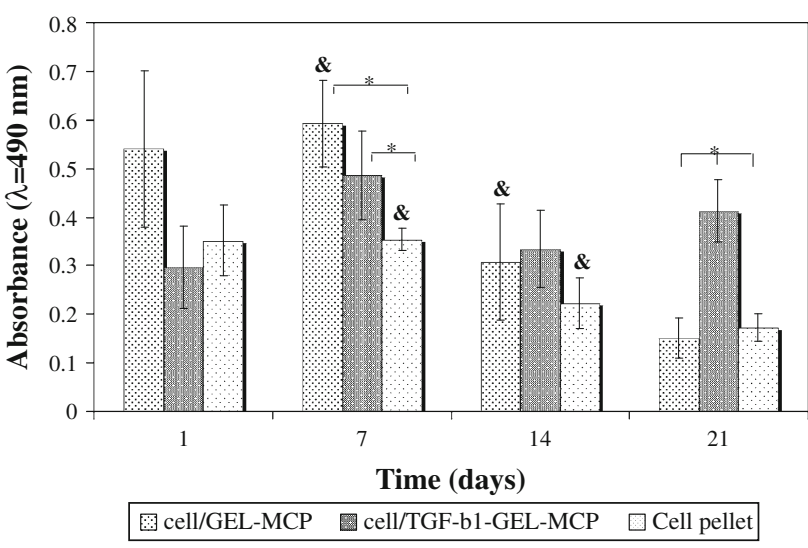

Fig. 4 MTS assay for cell/gelatin microparticle pellets (with and without TGF- $\beta 1$ ) and cell pellets, used as reference. Asterisks denote significant differences between samples on the same culture day. Ampersand represent significant differences on different culture days. Error bars represent means \pm SD with $n=4$

where approximately $24 \%$ of growth factor was released, followed by a slower release for the remaining 14 days. The cumulative release was $50 \%$ of initial loading by day 14 .

\subsection{Chondrocytes and Gel-MCP (with and without TGF- $\beta 1$ ) cultured in pellet}

The cell viability of chondrocytes seeded in crosslinked Gel-MCP with and without TGF- $\beta 1$ was examined by MTS assay. The obtained results after $24 \mathrm{~h}$ and 7, 14 and 21 days of culture show a consistent viability of the cell agglomerated with both, loaded and unloaded Gel-MCP (Fig. 4). Comparing the pellet of Gel-MCP with TGF- $\beta 1$ and the cell pellet (used in this case as control sample), the cell viability was improved by the TGF- $\beta 1$ released in the culture medium. That is way the number of viable chondrocytes remains almost constant after 21 days in the case of TGF- $\beta 1$ loaded Gel-MCP while viability after 1 week significantly decreased in both, unloaded Gel-MCP pellet and in the cell pellet.

Chondrocyte morphology evaluated by SEM of both cell/microparticle pellets (microparticles with and without TGF- $\beta 1$ ) and the control (cell pellet) (see Fig. 5) demonstrated that after 1 and 7 days of culture, the cells adhered very well on the surface of both types of microparticles (with and without TGF- $\beta 1$ ), forming interparticle cellular bridges (indicated by white arrows). Cells have a round morphology and can be distinguished from Gel-MCP by the characteristic surface roughness. As a result, microparticles were bound together. A homogeneous layer of chondrocytes, microparticles and ECM can be seen after 14 days of culture, in cell-TGF- $\beta 1$ loaded microparticle pellets. Some isolated cells emerge, with round morphology (Fig. 5). Cell aggregates were also observed in the constructs made with microparticles without TGF- $\beta 1$. The chondrocyte pellets revealed an elongated morphology after 14 days of culture in chondrogenic medium.

The expression of type I collagen and aggrecans and the actin cytoskeleton formation were examined by confocal laser scanning microscopy (CLSM). Cell/Gel-MCP pellets with and without TGF- $\beta 1$ and cell pellets (reference) were cross-sectioned in thick slices $(\sim 2 \mathrm{~mm})$. CLSM images after 14 and 21 days of culture are represented in Fig. 6. Nuclei were counterstained with DAPI (blue) and the expression of type I collagen and aggrecans was followed by Alexa-488 (green) and 647 (red) linked to the secondary antibody respectively. Positive expression of aggrecans (red) was observed mainly in the cell pellet (control material) and in the lesser extent in TGF- $\beta 1$ loaded GelMCP pellet, localized around the cells after 14 days. After 21 days of culture, the aggrecans expression was positive for cell/Gel-MCP (with and without TGF- $\beta 1$ ) pellet and cell pellet. In chondrocyte pellets, used as control, the secretion of type I collagen was clearly detected and predominantly on the surface of the cell pellet. On the contrary, in the cell/Gel-MCP (with and without TGF- $\beta 1$ ) only a few cells, even after 21 days, showed a positive staining.

Actin cytoskeleton was clearly detected in the cell pellets after 14 and 21 days of culture in chondrogenic medium compared to the cell/Gel-MCP pellets, where only in some cells was observed.

Glycosaminoglycans and type II collagen are the most important components of ECM in native articular cartilage; their expression in $3 \mathrm{D}$ culture is a recognized criterion of chondrogenecity. The results obtained in our constructs are represented in Figs. 7 and 8 respectively. The differences in GAG synthesis among the different supports are not significant at 14 days, nevertheless at 21 days of culture the amount of GAG per $20 \mathrm{mg}$ of Gel-MCP is significantly 

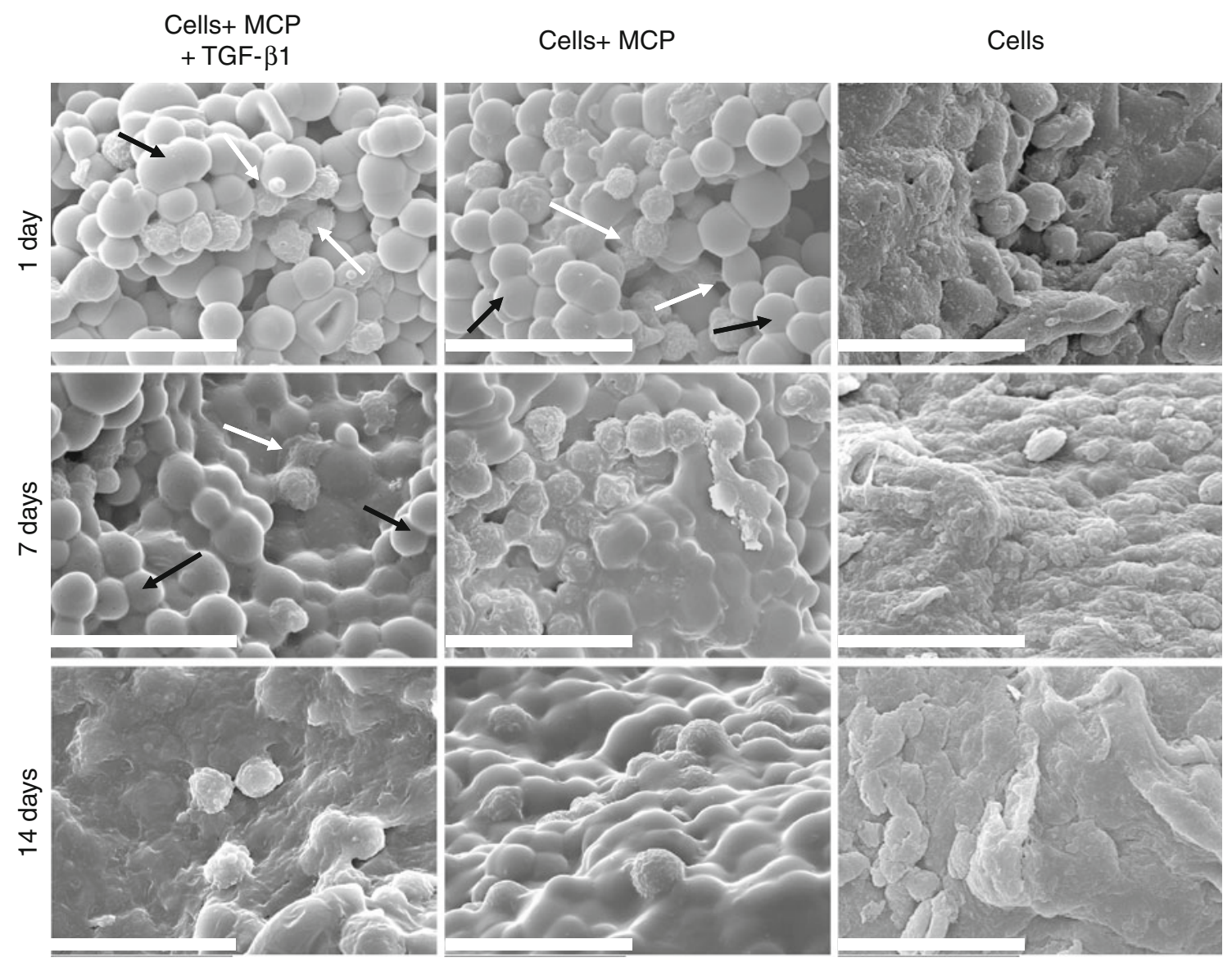

Fig. 5 Scanning electron microscopy images of chondrocytes seeded in TGF- $\beta 1$ loaded Gel-MCP (left column), control sample (microparticles without TGF- $\beta 1$ ) and cell pellet (right column) after 1, 7 and
14 days. Scale bars correspond to $40 \mu \mathrm{m}$. The white arrows indicate interparticle cellular bridges. The black arrows indicate the Gel-MCP higher in loaded microparticles than in unloaded microparticles or in the pellet.

Type II collagen synthesis was significantly increased from 14 to 21 days $(P<0.05)$ for cell/TGF- $\beta 1$ loaded GelMCP pellets being significantly higher than in the pellets or in unloaded Gel-MCP.

In cell pellets, GAG content clearly decreases with the culture time, while type II collagen deposition increase. This feature could be due to a partial dissolution of GAG as result of decrease of pellet size [21].

\section{Discussion}

Regeneration of articular cartilage with the technique of autologous chondrocyte implant, ACI, has proved to be able to produce newly formed cartilage tissue in the region of the cartilage defect although the evolution of the tissue with time present important drawbacks including the degeneration of the tissue to fibrocartilage with poor mechanical properties [22-24]. The implant of a mature chondrocyte pellet in the cartilage defect in a rabbit model also produced a cartilaginous tissue lacking the organization of hyaline cartilage and with very low elastic modulus after 3 months of implantation [25]. Culture of chondrocytes in a pellet in chondrogenic medium produce cells with round morphology with production of ECM with abundance of glycosaminoglycans and type II collagen, but expressing negative markers for hyaline cartilage chondrocytes as type I collagen [26-28]. The presence of a scaffolding system that supports cell adhesion and controls stress transmission to the cells can improve the performance of chondrocyte transplant to a cartilage defect. The strategy proposed in this work is somewhere in between the entrapping of chondrocytes in a gel such as alginate [29], poly (ethylene oxide) [30], oligo poly(ethylene glycol) fumarate [11, 31], poly (ethylene glycol dimethacrylate) [32, 33], silk [34] extensively explored in the literature and seeding the cells in a macroporous scaffold in which they adhere to the pore walls a situation which is not far from monolayer culture. The cell-microparticles suspension can be injected in the zone of the cartilage following the 


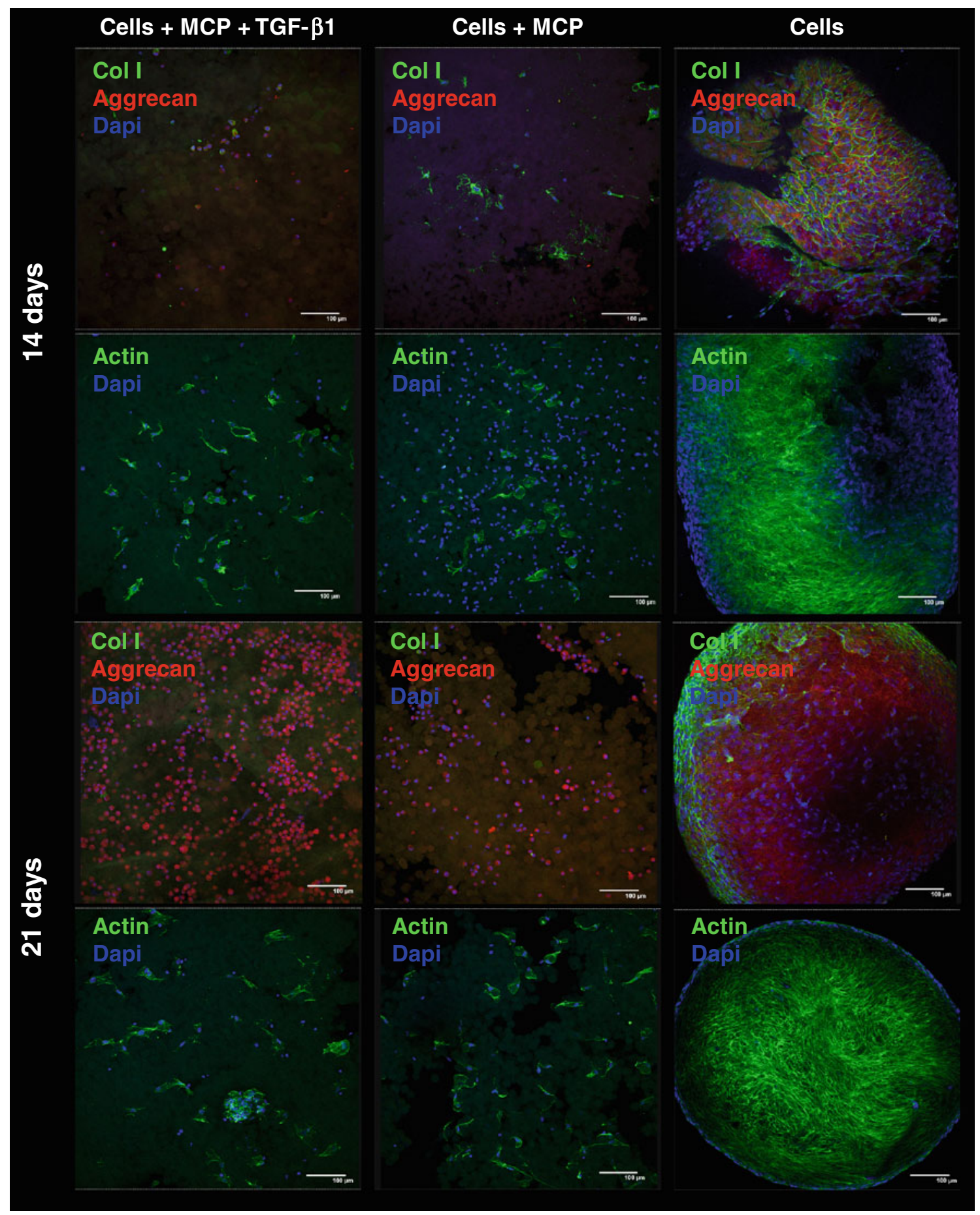

Fig. 6 Actin cytoskeleton (green) development and immunofluorescence staining for aggrecan (red) and type I collagen (green) of chondrocytes/gelatin microparticle pellets (with and without TGF- $\beta 1$ )

surgical approaches currently used in ACI practice, but adding the required support for cell attachment.

In this study, we crosslinked Gel-MCP with genipin, which was reported to be non cytotoxic and a safe crosslinking agent, compared to commonly used glutaraldehyde or formaldehyde crosslinkers [35]. Nevertheless, Wang and cell pellets cultured after 14 and 21 days in chondrogenic medium. The scale bar represents $100 \mu \mathrm{m}$ for all images. The cells nuclei were counterstained with DAPI (blue) (Color figure online)

et al. [36] recently reported a certain toxicity of genipin on chondrocytes and osteoblasts at high doses being its toxicity depended on doses but not on the time. Genipin has been used as a culture medium supplement to develop enhanced engineered cartilage [37]. Chitosan microparticles crosslinked with genipin were also used to culture 


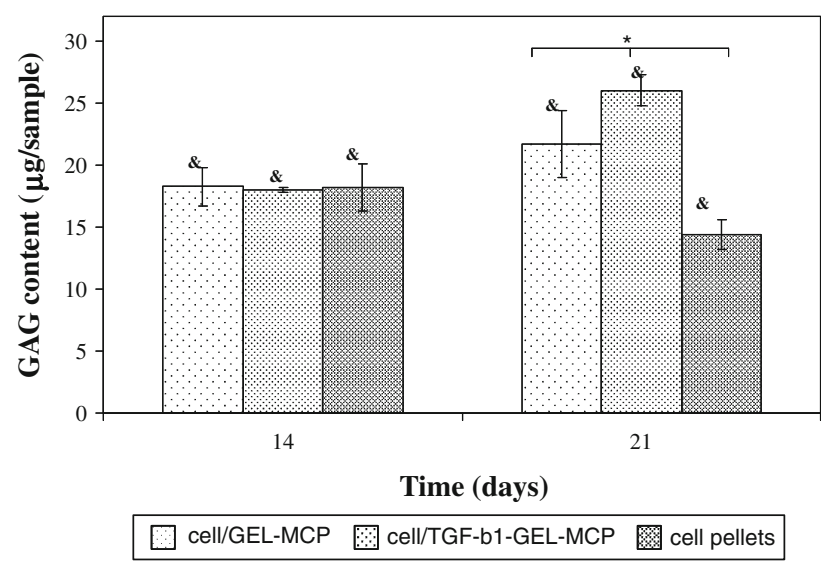

Fig. 7 GAG quantification assay after chondrocyte/gelatin microparticle pellets (with and without TGF- $\beta 1$ ) and cell pellets cultured for 2 and 3 weeks in chondrogenic medium. Asterisks denote significant differences between samples on the same culture day. Ampersand represent significant differences on different culture days. Error bars represent means $\pm \mathrm{SD}$ with $n=5$

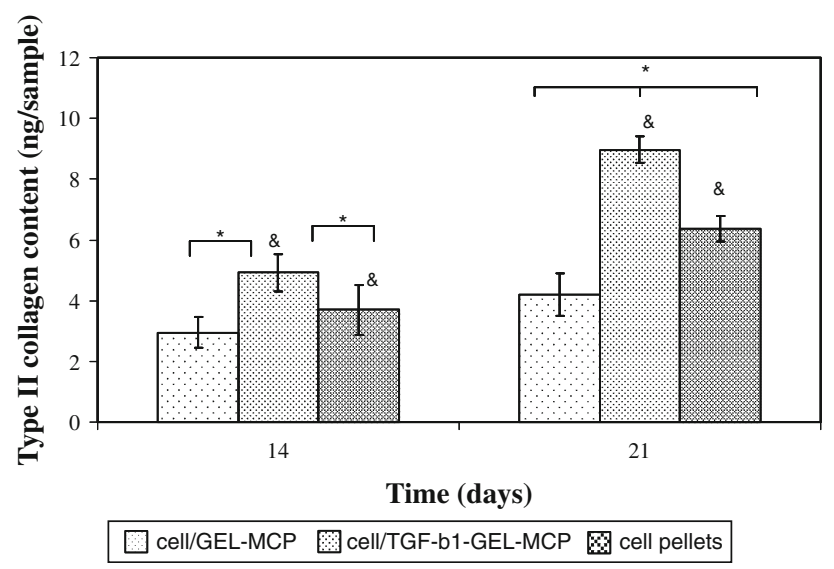

Fig. 8 Collagen II content after chondrocyte/gelatin microparticle pellets (with and without TGF- $\beta 1$ ) and cell pellets cultured for 2 and 3 weeks in chondrogenic medium. Asterisks denote significant differences between samples on the same culture day. Ampersand represent significant differences on different culture days. Error bars represent means \pm SD with $n=5$

mesenchymal stem cells in osteogenic medium during 14 days, showing no signs of toxicity [18]. In addition, the results published by Solorio et al. [38] demonstrated the potential of Gel-MCP crosslinked with genipin to deliver growth factors locally to the cells.

The crosslinking reaction during the formation of microparticles results in a $26 \%$ crosslinking degree. Crosslinking of already formed Gel-MCP can yield higher crosslinking density as reported in the $[39,40]$. The strategy of this work was to prepare the water/oil emulsion in PBS (pH 7.4) at $37{ }^{\circ} \mathrm{C}$ using a mixture of biopolymer and growth factor as aqueous phase. In this way an ionic complex is formed between the acidic gelatin (isoelectric point 5.0) and TGF- $\beta 1$ (isoelectric point 9.5) [41] to which genipin is added. It can be expected that some of the growth factor molecules be linked to gelatin by covalent bonds created by the crosslinker.

At 14 days the cumulative release of TGF- $\beta 1$ is around $50 \%$ (Fig. 3). Growth factor molecules fixed to the matrix by covalent bonds would be delivered only by support degradation at longer times. The significant differences in chondrocyte response between TGF- $\beta 1$ loaded and unloaded microparticles supports that delivered growth factor is bioactive.

Recent studies by Catela et al. [42] demonstrated that TGF- $\beta 1$ released from fibrin gels to which was covalently bonded was bioactive inducing chondrogenic differentiation of mesenchymal stem cells. Crosslinking considerably reduce water uptake of Gel-MCP and improves their stability in aqueous medium. Crosslinking density can be used to modulate the rate of sustained delivery of TGF- $\beta 1$.

In this work we have shown that Gel-MCP agglomerated together with the expanded chondrocytes create a 3D environment, without the need of another gel embedding both Gel-MCP and cells as proposed in other works [10-12]. The construct is consistent and easily handleable, due to the strong adhesion between the ECM produced by the cells and the Gel-MCP. Interestingly enough, due to the Gel-MCP/cells ratio these microparticles acts as a 3D scaffold, favouring cell to matrix interaction with respect to cell to cell contacts and even, without growth factor supply, the behaviour of the seeded chondrocytes change significantly with respect to the cell pellet: increased cell viability, sharp diminution of type I collagen in the produced ECM, absence of actin stress fibers in most of the cells, and increased production of aggrecan. On the other hand, GelMCP may act as a mean for stress transmission to the cells. Acquisition and maintenance of the chondrocyte phenotype "in vivo" is highly addressed by dynamic compression loading to which articular cartilage is subjected. The transmission of these stresses to the cells in a pellet is quite different to the situation in healthy cartilage where cells are isolated in lacunae and protected by a quite hard tissue. Crosslinked Gel-MCP can be produced with an elastic modulus that can vary in a broad range, thus, it allows designing the best conditions to create the adequate environment with respect to compression load sustaining. On the other hand, we have shown that sustained delivery of TGF- $\beta 1$ can be obtained from the cell/support agglomerate itself, thus low amounts of the growth factor can be delivered to the cells locally with small dissemination to the rest of the organism. The improvement of cell behaviour in the culture with TGF- $\beta 1$ delivery is very clear, with increased viability and chondrogenic markers with respect to the culture in non-loaded microspheres. 


\section{Conclusions}

Cell/Gel-MCP constructs allow culture and redifferentiation of mature chondrocytes previously expanded in monolayer culture. Cell adhesion to microparticles agglomerates the whole construct, which from the first day of culture maintains coherence and can be easily handled. TGF- $\beta 1$ can be effectively included in the Gel-MCP during their preparation by a water-oil emulsion process. Crosslinking with genipin allows progressive delivery when the particles are immersed in culture medium. Chondrocytes culture in agglomerates with growth factor delivery show viability for longer times, increased production of ECM components characteristics of hyaline cartilage. The results obtained indicate that the developed gelatin-based microparticles could be used as an injectable scaffold to delivery chondrocytes through minimally invasive procedures, that will agglomerate in situ by the action of the cells; the particles are be able to provide mechanical and a 3D structural support for the cells and also act as a system able to release relevant bioactive molecules.

Acknowledgments JLGR acknowledge the support of the Spanish Ministry of Education through project No. MAT2010-21611-C03-01 (including the FEDER financial support). The support of the Instituto de Salud Carlos III (ISCIII) through the CIBER initiative of the Networking Research Center on Bioengineering, Biomaterials and Nanomedicine (CIBER-BBN) is also acknowledged.

\section{References}

1. Danisovic L, Varga I, Zamborsky R, Bohmer D. The tissue engineering of articular cartilage: cells, scaffolds and stimulating factors. Exp Biol Med. 2012;237(1):10-7.

2. Darling EM, Athanasiou KA. Rapid phenotypic changes in passaged articular chondrocyte subpopulations. J Orthop Res. 2005;23:425-32.

3. Oliveira MB, Mano JF. Polymer-based microparticles in tissue engineering and regenerative medicine. Biotechnol Prog. 2011; 27(4):897-912.

4. Zhang R, Xue M, Yang J, Tan T. A novel injectable and in situ crosslinked hydrogel based on hyaluronic acid and $\alpha, \beta$-polyaspartylhydrazide. J Appl Polym Sci. 2012;125(2):1116-26.

5. Hou Q, Chau DYS, Pratoomsoot C, Tighe PJ, Dua HS, Shakesheff $\mathrm{KM}$, et al. In situ gelling hydrogels incorporating microparticles as drug delivery carriers for regenerative medicine. J Pharm Sci. 2008;97(9):3972-80.

6. Singh A, Suri S, Roy K. In situ crosslinking hydrogels for combinatorial delivery of chemokines and siRNA-DNA carrying microparticles to dendritic cells. Biomaterials. 2009;30(28): 5187-200.

7. Bidarra SJ, Barrias CC, Fonseca KB, Barbosa MA, Soares RA, Granja PL. Injectable in situ crosslinkable RGD-modified alginate matrix for endothelial cells delivery. Biomaterials. 2011; 32(31):7897-904.

8. Zheng Shu X, Liu Y, Palumbo FS, Luo Y, Prestwich GD. In situ crosslinkable hyaluronan hydrogels for tissue engineering. Biomaterials. 2004;25(7-8):1339-48.
9. Fan HB, Zhang CL, Li J, Bi L, Qin L, Wu H, et al. Gelatin microspheres containing TGF-beta 3 enhance the chondrogenesis of mesenchymal stem cells in modified pellet culture. Biomacromolecules. 2008;9(3):927-34.

10. Han YS, Wei YY, Wang SS, Song Y. Cartilage regeneration using adipose-derived stem cells and the controlled-released hybrid microspheres. Jt Bone Spine. 2010;77(1):27-31.

11. Park H, Temenoff JS, Tabata Y, Caplan AI, Mikos AG. Injectable biodegradable hydrogel composites for rabbit marrow mesenchymal stem cell and growth factor delivery for cartilage tissue engineering. Biomaterials. 2007;28(21):3217-27.

12. Hu XH, Zhou J, Zhang N, Tan HP, Gao CY. Preparation and properties of an injectable scaffold of poly(lactic-co-glycolic acid) microparticles/chitosan hydrogel. J Mech Behav Biomed Mater. 2008;1(4):352-9.

13. Tan HP, Chu CR, Payne KA, Marra KG. Injectable in situ forming biodegradable chitosan-hyaluronic acid based hydrogels for cartilage tissue engineering. Biomaterials. 2009;30(13): 2499-506.

14. Malda J, Kreijveld E, Temenoff JS, van Blitterswijk CA, Riesle J. Expansion of human nasal chondrocytes on macroporous microcarriers enhances redifferentiation. Biomaterials. 2003;24(28): 5153-61.

15. Glattauer V, White JF, Tsai WB, Tsai CC, Tebb TA, Danon SJ, et al. Preparation of resorbable collagen-based beads for direct use in tissue engineering and cell therapy applications. J Biomed Mater Res Part A. 2010;92A(4):1301-9.

16. Pettersson S, Wettero J, Tengvall P, Kratz G. Human articular chondrocytes on macroporous gelatin microcarriers form structurally stable constructs with blood-derived biological glues in vitro. J Tissue Eng Regen Med. 2009;3(6):450-60.

17. Fan HB, Hu YY, Qin L, Li XS, Wu H, Lv R. Porous gelatinchondroitin-hyaluronate tri-copolymer scaffold containing microspheres loaded with TGF-beta 1 induces differentiation of mesenchymal stem cells in vivo for enhancing cartilage repair. J Biomed Mater Res Part A. 2006;77A(4):785-94.

18. García Cruz DM, Escobar Ivirico JL, Gomes MM, Gómez Ribelles JL, Sánchez, Reis RL, et al. Chitosan microparticles as injectable scaffolds for tissue engineering. J Tissue Eng Regen Med. 2008;2(6):378-80.

19. Leane MM, Nankervis R, Smith A, Illum L. Use of the ninhydrin assay to measure the release of chitosan from oral solid dosage forms. Int J Pharm. 2004;271(1-2):241-9.

20. Pérez Olmedilla M, Garcia-Giralt N, Pradas MM, Ruiz PB, Gómez Ribelles JL, Palou EC, et al. Response of human chondrocytes to a non-uniform distribution of hydrophilic domains on poly (ethyl acrylate-co-hydroxyethyl methacrylate) copolymers. Biomaterials. 2006;27(7):1003-12.

21. Alves da Silva ML, Crawford A, Mundy JM, Correlo VM, Sol P, Bhattacharya $\mathrm{M}$, et al. Chitosan/polyester-based scaffolds for cartilage tissue engineering: Assessment of extracellular matrix formation. Acta Biomaterialia. 2010;6(3):1149-57.

22. Smith GD, Knutsen G, Richardson JB. A clinical review of cartilage repair techniques. J Bone Jt Surg Br Vol. 2005;87B(4): $445-9$.

23. Nehrer S, Domayer S, Dorotka R, Schatz K, Bindreiter U, Kotz $\mathrm{R}$. Three-year clinical outcome after chondrocyte transplantation using a hyaluronan matrix for cartilage repair. Eur J Radiol. 2006;57(1):3-8.

24. Brittberg M, Peterson L, Sjogren-Jansson E, Tallheden T, Lindahl A. Articular cartilage engineering with autologous chondrocyte transplantation-A review of recent developments. J Bone Jt Surg Am Vol. 2003;85A:109-15.

25. Martinez-Diaz S, Garcia-Giralt N, Lebourg M, Gomez-Tejedor JA, Vila G, Caceres E, et al. In vivo evaluation of 3-dimensional 
polycaprolactone scaffolds for cartilage repair in rabbits. Am J Sports Med. 2010;38(3):509-19.

26. Pfander D, Rahmanzadeh R, Scheller EE. Presence and distribution of collagen II, collagen I, fibronectin, and tenascin in rabbit normal and osteoarthritic cartilage. J Rheumatol. 1999;26: 386-94.

27. Huch K, Mordstein V, Stove J, Nerlich AG, Arnholdt H, Delling $\mathrm{G}$, et al. Expression of collagen type I, II, X and Ki-67 in osteochondroma compared to human growth plate cartilage. Eur J Histochem. 2002;46(3):249-58.

28. Gohring AR, Lubke C, Andreas K, Haupl T, Sittinger M, Ringe J, et al. Tissue-engineered cartilage of porcine and human origin as in vitro test system in arthritis research. Biotechnol Prog. 2010;26(4):1116-25.

29. Tritz J, Rahouadj R, de Isla N, Charif N, Pinzano A, Mainard D, et al. Designing a three-dimensional alginate hydrogel by spraying method for cartilage tissue engineering. Soft Matter. 2010;6(20):5165-74.

30. Elisseeff J, McIntosh W, Fu K, Blunk T, Langer R. Controlledrelease of IGF-I and TGF- $\beta 1$ in a photopolymerizing hydrogel for cartilage tissue engineering. J Orthop Res. 2001;19(6):1098-104.

31. Park H, Temenoff JS, Holland TA, Tabata Y, Mikos AG. Delivery of TGF-beta 1 and chondrocytes via injectable, biodegradable hydrogels for cartilage tissue engineering applications. Biomaterials. 2005;26(34):7095-103. doi:10.1016/j.biomaterials. 2005.05.083.

32. Hwang NS, Varghese S, Zhang Z, Elisseeff J. Chondrogenic differentiation of human embryonic stem celīDerived cells in arginine-glycine-aspartatēModified hydrogels. Tissue Eng. 2006;12(9):2695-706.

33. Riley SL, Dutt S, de la Torre R, Chen AC, Sah RL, Ratcliffe A. Formulation of PEG-based hydrogels affects tissue-engineered cartilage construct characteristics. J Mater Sci Mater Med. 2001;12(10):983-90.
34. Chao P-HG, Yodmuang S, Wang X, Sun L, Kaplan DL, VunjakNovakovic G. Silk hydrogel for cartilage tissue engineering. J Biomed Mater Res B Appl Biomater. 2010;95B(1):84-90.

35. Nishi C, Nakajima N, Ikada Y. In vitro evaluation of cytotoxicity of diepoxy compounds used for biomaterial modification. J Biomed Mater Res. 1995;29(7):829-34.

36. Wang C, Lau TT, Loh WL, Su K, Wang D-A. Cytocompatibility study of a natural biomaterial crosslinker-Genipin with therapeutic model cells. J Biomed Mater Res B Appl Biomater. 2011; 97B(1):58-65.

37. Lima EG, Tan AR, Tai T, Marra KG, DeFail A, Ateshian GA, et al. Genipin enhances the mechanical properties of tissueengineered cartilage and protects against inflammatory degradation when used as a medium supplement. J Biomed Mater Res Part A. 2009;91A(3):692-700.

38. Solorio L, Zwolinski C, Lund AW, Farrell MJ, Stegemann JP. Gelatin microspheres crosslinked with genipin for local delivery of growth factors. J Tissue Eng Regen Med. 2010;4(7):514-23.

39. Lau TT, Wang C, Wang D-A. Cell delivery with genipin crosslinked gelatin microspheres in hydrogel/microcarrier composite. Compos Sci Technol. 2010;70(13):1909-14.

40. Solorio LD, Vieregge EL, Dhami CD, Dang PN, Alsberg E. Engineered cartilage via self-assembled hMSC sheets with incorporated biodegradable gelatin microspheres releasing transforming growth factor-b1. J Controlled Release. 2012;158(2): 224-32.

41. Yamamoto M, Ikada Y, Tabata Y. Controlled release of growth factors based on biodegradation of gelatin hydrogel. J Biomater Sci Polym Ed. 2001;12(1):77-88.

42. Catelas I, Dwyer JF, Helgerson S. Controlled release of bioactive transforming growth factor beta- 1 from fibrin gels in vitro. Tissue Eng Part C Method. 2008;14(2):119-28. 